\title{
Extending the Regge description of hadronic forward scattering from the multi-TeV down to the GeV region
}

\author{
Jose R. Pelaez ${ }^{* \dagger}$ \\ Departamento de Física Teórica II, Universidad Complutense, 28040, Madrid, Spain \\ E-mail: jrpelaez@fis.ucm.es
}

\begin{abstract}
We provide a simple Regge parametrization of forward hadronic scattering from the multi-TeV range down to $\sim 1 \mathrm{GeV}$ above the threshold of each reaction. We show how, at these relatively low energies, mass effects are relevant and should be properly taken into account, and that the data favor a logarithmic growth of the Pomeron contribution that is based on an improved unitarity bound at intermediate energies as well as a separate factorization of the singularities. Data on both the imaginary and real parts of amplitudes are remarkably well described with this parametrization. Here we also show that the description does not depend on the strategy adopted to include or not systematic uncertainties in different data sets.
\end{abstract}

International Europhysics Conference on High Energy Physics

July 21 st - 27th 2005

Lisboa, Portugal

${ }^{*}$ Speaker.

${ }^{\dagger}$ Work partially supported by CGICYT Spain, under contracts FPA2005-02327 and BFM2002-01868 and the EURIDICE network contract HPRN-CT-2002-00311, as well as the EU Hadron Physics Project, contract number RII3-CT2004-506078. 
Recently [1] we extended down to $\sim 1 \mathrm{GeV}$ above threshold the Regge description of $(\bar{p} p+$ $p p),\left(K^{+} p+K^{-} p\right), \pi^{ \pm} N$ and $\pi \pi$ cross sections, which only involve the Pomeron, $f$ (or $\left.P^{\prime}\right)$ and $\rho$ trajectories. I present here preliminary results [2] adding also the $a$ and $\omega$ trajectories and extending the analysis to $\bar{p} p, p p, \bar{p} n, p n, K^{ \pm} p, K^{ \pm} n, \pi^{ \pm} N$ and $\pi \pi$ total cross sections, and $I m F / R e F$ ratios for the $\bar{p} p, p p, p n, \pi^{ \pm} N$ and $K^{ \pm} p$ forward elastic amplitudes, $F$. The data come from the COMPAS group compilation. However, the original references did not treat systematic uncertainties uniformly, and many data sets are incompatible within their statistical errors, and hence cannot be described simultaneously. Thus, we adopt two fitting strategies: First, we keep the original uncertainties so that we can easily compare with the PDG [3] and the reference works of the COMPETE group [4]. However, this introduces an artificially large $\chi^{2} / d$.o.f. and a bias toward those data sets that do not provide systematic uncertainties. Hence, in our second strategy, we add a systematic error, but only to those data without it, of $0.5 \%$ for $p p, 1 \%$ for $\bar{p} p$ and $1.5 \%$ for other processes. These additional errors are similar to those given by other experiments, and thus all sets are equally weighted. To account for different ways of combining statistical and systematic errors, in the first strategy we have added them in quadrature and linearly in the second. In addition, we use $\sigma^{\text {total }}$ data [5] on $\pi^{+} \pi^{-}, \pi^{-} \pi^{-}, \pi^{+} \pi^{0}$, above $1.42 \mathrm{GeV}$, plus one data point per channel reconstructed from phase shift analyses [1] at $1.42 \mathrm{GeV}$. If using $\pi \pi$ low energy information [1, 2], the $\rho$ residue and the intercept come out somewhat smaller and larger, respectively.

The different Regge trajectories contribute to the amplitudes as follows:

$$
\begin{array}{r}
F_{p^{ \pm} p}=\left(P_{N N}+f_{N N}+a_{N N} \mp \omega_{N N} \mp \rho_{N N}\right) / 2, F_{p^{ \pm} n}=\left(P_{N N}+f_{N N}-a_{N N} \mp \omega_{N N} \pm \rho_{N N}\right) / 2, \\
F_{K^{ \pm} p}=\left(P_{K N}+f_{K N}+a_{K N} \mp \omega_{K N} \mp \rho_{K N}\right) / 2, F_{K^{ \pm} n}=\left(P_{K N}+f_{K N}-a_{K N} \mp \omega_{K N} \pm \rho_{K N}\right) / 2, \\
F_{\pi^{ \pm} p}=\left(P_{\pi N}+f_{\pi N}\right) / \sqrt{6} \mp \rho_{\pi N} / 2, \quad F_{\pi^{ \pm} \pi^{-}}=\left(P_{\pi \pi}+f_{\pi \pi}\right) / 3 \pm \rho_{\pi \pi} / 2, \quad F_{\pi^{0} \pi^{-}}=\left(P_{\pi \pi}+f_{\pi \pi}\right) / 3,
\end{array}
$$

where $N=p^{ \pm}, n$ and we use the factorization [6] relations $R_{A B}(v)=f_{A}^{R} f_{B}^{R} R(v)$, with

$$
R(v)=\beta_{R}\left(\frac{1+\tau e^{-i \pi \alpha}}{\sin \pi \alpha}\right) v^{\alpha_{R}}, \quad \text { for } R=\rho, f, a, \omega
$$

where $\tau$ is the signature of the trajectory. Masses are correctly taken into account by using the Regge variable $v=(s-u) / 2$, which, for forward scattering, is $v=s-m_{a}^{2}-m_{b}^{2}>s-s_{t}$. Following the QCD version of Regge theory, and the recent analysis [4], we assume $\alpha_{a}=\alpha_{f}$ and $\alpha_{\omega}=\alpha_{\rho}$. We set $f_{\pi}^{R}=1$, for $R=P, f, \rho$ and $\beta_{R}=1$ for $R=a, \omega$, since they are redundant.

For the Pomeron, we propose the use of a "constant plus logarithm" law, i.e.,

$$
P_{A B}=C_{A B}+L_{A B}, \quad \operatorname{Im} P(v)=v\left(\beta_{P}+A \log ^{2}\left[\frac{v-v_{t h}}{v_{1} \log ^{7 / 2}\left(v / v_{2}\right)}\right]\right),
$$

where $v_{t h}$ is the right-cut branch point of each amplitude. This law follows the improved unitarity bound in [7] that grows faster than $s \log s$ but slower at intermediate energies than the $s \log ^{2} s$ Froissart bound, which is recovered at very high $s$. The generalized "factorization theorem" [8] requires singularities to factorize separately, and as a first approximation we thus use separated $f_{A}^{C}$ and $f_{A}^{L}$.

Finally, the $\operatorname{Re} F$ are obtained from dispersive representations, and total cross sections from:

$$
\sigma_{a b}=4 \pi^{2} \operatorname{Im} F_{a+b \rightarrow a+b}(s, 0) / \lambda^{1 / 2}\left(s, m_{a}^{2}, m_{b}^{2}\right), \quad \lambda\left(s, m_{a}^{2}, m_{b}^{2}\right)=s^{2}+\left(m_{a}^{2}-m_{b}^{2}\right)^{2}-2 s\left(m_{a}^{2}+m_{b}^{2}\right) .
$$


Let us remark that $\lambda$ is usually approximated by $s^{2}$, although very recently [9] a slight improvement in $\chi^{2} /$ d.o.f. has been reported using $\lambda$, instead of $s^{2}$, down to $\sqrt{s}=5 \mathrm{GeV}$. Note that, when $E_{k i n} \simeq 1 \mathrm{GeV}$, as in our case here, the use of $s^{2}$, instead of $\lambda$, yields a $30 \%$ overestimation for $N N$.

Different sets of parameters from fits to data using the two strategies described above are shown in Table 1, including the uncertainty obtained from the $\chi^{2} /$ d.o.f. minimization (using MINUIT), for strategy 2 , whose $\chi^{2} /$ d.o.f. $=0.85$ for 1186 data points. Due to the strong correlations, amplitudes should be calculated using with parameters within the same set. However, systematic errors for a single parameters can be estimated from the difference between strategies.

\begin{tabular}{|c|c|c|c|c|c|c|c|}
\hline$E_{\text {kin }}^{\min }$ & $\begin{array}{l}\text { strategy } 2 \\
1-1.3 \mathrm{GeV}\end{array}$ & $\begin{array}{c}\text { strategy } 1 \\
1-1.3 \mathrm{GeV} \\
\end{array}$ & $\begin{array}{l}\text { Minuit } \\
\text { errors }\end{array}$ & $E_{\text {kin }}^{\min }$ & $\begin{array}{l}\text { strategy } 2 \\
1-1.3 \mathrm{GeV}\end{array}$ & $\begin{array}{l}\text { strategy } 1 \\
1-1.3 \mathrm{GeV}\end{array}$ & $\begin{array}{l}\text { Minuit } \\
\text { errors }\end{array}$ \\
\hline$\beta_{P}$ & 0.746 & 0.937 & 0.003 & $f_{K}^{f}$ & 0.30 & 0.32 & 0.01 \\
\hline$f_{N}^{P}$ & 1.792 & 1.705 & 0.007 & $\alpha_{f}$ & 0.646 & 0.640 & 0.002 \\
\hline$f_{K}^{P}$ & 0.731 & 0.714 & 0.004 & $f_{N}^{a}$ & -0.24 & 0.25 & 0.04 \\
\hline$A$ & 0.043 & 0.050 & 0.001 & $f_{K}^{a}$ & -0.55 & 0.5 & 0.1 \\
\hline$v_{1}$ & 0.0005 & 0.001 & 0.0001 & $\beta_{\rho}$ & 1.28 & 1.34 & 0.11 \\
\hline$v_{2}$ & 0.676 & 0.633 & 0.001 & $f_{N}^{\rho}$ & 0.51 & 0.46 & 0.04 \\
\hline$f_{N}^{l o g}$ & 1.02 & 0.993 & 0.001 & $f_{K}^{\rho}$ & 0.49 & 0.54 & 0.04 \\
\hline$f_{K}^{l o g}$ & 0.723 & 0.733 & 0.012 & $\alpha_{\rho}$ & 0.464 & 0.464 & 0.003 \\
\hline$\beta_{f}$ & 1.70 & 1.77 & 0.014 & $f_{N}^{\omega}$ & 1.97 & 1.98 & 0.015 \\
\hline$f_{N}^{f}$ & 1.78 & 1.75 & 0.01 & $f_{K}^{\omega}$ & 0.66 & 0.65 & 0.01 \\
\hline & & & & $\sigma_{L H C}$ & $109 \mathrm{mb}$ & $110 \mathrm{mb}$ & $1 \mathrm{mb}$ \\
\hline
\end{tabular}

Table 1. Fit parameters with different strategies. The Minuit errors are just statistical, and nominal, since the parameters are strongly correlated and can only be used with the central values of strategy 2.

In Table 2 we show how the $\chi^{2} /$ d.o.f. deteriorates if we do not implement one of the following items: i) using $v$ instead of $s$, ii) the logarithmic growth in Eq.(2), iii) the separate factorization.

\begin{tabular}{|c|c|c|c|c|}
\hline$E_{\text {kin }}^{\min }(\mathrm{GeV})$ & $1-1.3$ & 1.5 & 2 & 3 \\
\hline \# data points & 1186 & 1002 & 895 & 768 \\
\hline \hline Parametrization & \multicolumn{5}{|c|}{$\chi^{2} /$ d.o.f. for strategy $2 / 1$} \\
\hline Ours & $0.85 / 1.56$ & $0.63 / 1.14$ & $0.57 / 1.05$ & $0.52 / 0.95$ \\
\hline$v_{1} \equiv 0.01 \mathrm{GeV}^{2}$ & $0.85 / 1.57$ & $0.63 / 1.26$ & $0.58 / 1.06$ & $0.52 / 0.97$ \\
\hline \hline powers of $s^{\alpha}$ & $1.58 / 2.87$ & $1.16 / 2.11$ & $0.99 / 1.80$ & $0.78 / 1.42$ \\
\hline \hline \multicolumn{5}{|c|}{ Pomeron logarithmic term } \\
\hline$v \log (v)$ & $1.01 / 1.83$ & $0.69 / 1.26$ & $0.59 / 1.09$ & $0.52 / 0.97$ \\
\hline$v \log \left(v-v_{\text {th }}\right)$ & $1.03 / 1.83$ & $0.69 / 1.27$ & $0.59 / 1.12$ & $0.52 / 0.98$ \\
\hline$v \log ^{2}(v)$ & $0.97 / 1.79$ & $0.68 / 1.24$ & $0.59 / 1.10$ & $0.52 / 0.95$ \\
\hline$v \log ^{2}\left(v-v_{t h}\right)$ & $0.91 / 1.68$ & $0.65 / 1.18$ & $0.58 / 1.06$ & $0.52 / 0.95$ \\
\hline \hline \multicolumn{5}{|c|}{ Factorization of Pomeron logarithms } \\
\hline$f_{a}^{L} \equiv 1($ as PDG) & $0.92 / 1.70$ & $0.66 / 1.23$ & $0.59 / 1.10$ & $0.54 / 1.01$ \\
\hline$f_{a}^{C}=f_{a}^{L}$ & $0.89 / 1.67$ & $0.64 / 1.41$ & $0.60 / 1.14$ & $0.58 / 1.02$ \\
\hline
\end{tabular}

Table 2. $\chi^{2} /$ d.o.f. for several $E_{k i n}^{\min }$ and different modifications of our parametrization.

In [2], we have already shown in plots that our parametrization provides a remarkable description of total $N N, \pi \pi, \pi^{ \pm} N, K^{ \pm} p$ and $K^{ \pm} n$ cross sections and $R e F / \operatorname{Im} F$. The simple parametrization reported here was shown to describe remarkably well 20 observables extending from several $\mathrm{TeV}$ down to $\sim 1 \mathrm{GeV}$ above the threshold of each reaction. We will not repeat the plots here and instead we will show that, although the central values of each parameter for strategy 2 could be beyond 
one standard deviation from those of strategy 2 , when considering the complete parameter sets, the results overlap. Indeed, we show in Figure 1 the curves obtained from our parametrization from strategy 2 (the continuous line and gray bands covering its nominal uncertainties), versus the results for strategy 1 (the dashed line). Although we have chosen the plot where they deviate most, both curves are almost indistinguishable.

Further details will be given in a forthcoming publication [2]. We hope that, apart from establishing the logarithmic growth of the Pomeron, our parametrization could be easily used for dispersive studies in hadronic physics that involve integrals from the resonance region to infinity.

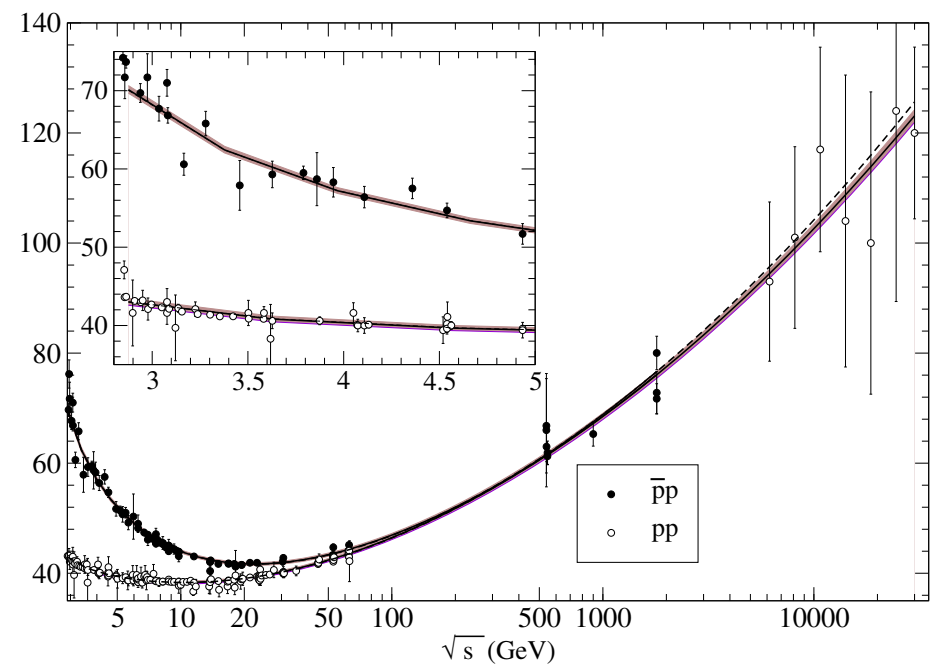

Figure 1: Total $p p$ and $\bar{p} p$ cross sections. Results from our parametrization down to $1 \mathrm{GeV}$ above threshold both for strategy 2 (continuous line) and strategy 1 (dashed line). Note that both curves basically overlap over the bands that cover the nominal uncertainties in the parameters. For other processes the agreement between strategies is even better.

\section{References}

[1] J. R. Peláez and F. J. Yndurain, Phys. Rev. D 68, 074005 (2003); Phys. Rev. D 69, 114001 (2004) and hep-ph/0411334.

[2] J.R. Pelaez, hep-ph/0510215 for preliminary results and also in preparation.

[3] The review of Particle Physics S. Eidelman et al., Phys. Lett. B592, 1 (2004). K. Hagiwara et al., Phys. Rev. D66, 010001 (2002). D.E. Groom et al, Eur. Phys. J. C15 (2000) 1.

[4] J. R. Cudell et al.,(COMPETE Collab.) Phys. Rev. D 65, 074024 (2002)

[5] Biswas, N. N., et al., Phys. Rev. Lett., 18, 273 (1967) Cohen, D. et al., Phys. Rev. D7, 661 (1973). Robertson, W. J., Walker, W. D., and Davis, J. L., Phys. Rev. D7, 2554 (1973). Hanlon, J., et al, Phys. Rev. Lett., 37, 967 (1976); Abramowicz, H., et al. Nucl. Phys., B166, 62 (1980)

[6] Gell-Mann, M. Phys. Rev. Lett., 8, 263, (1962); Gribov, V. N., and Pomeranchuk, I. Ya. Phys. Rev. Lett., 8, 343, (1962).

[7] F. J. Yndurain, Phys. Lett. B 41, 591 (1972). ( Note that $\log ^{7}$ was incorrectly given instead of $\log ^{7 / 2}$ ).

[8] J. R. Cudell, E. Martynov and G. Soyez, Nucl. Phys. B 682, 391 (2004)

[9] J. R. Cudell, E. Martynov, O. Selyugin and A. Lengyel, Phys. Lett. B 587, 78 (2004) 